\title{
Fermentation by gut microbiota cultured in a simulator of the human intestinal microbial ecosystem is improved by probiotic Enterococcus faecium CRL 183
}

\author{
Katia Sivieri $^{1}$, Fernanda Bianchi ${ }^{1}$, Maria A. Tallarico ${ }^{2}$, and Elizeu A. Rossi ${ }^{1}$ \\ ${ }^{1}$ Department of Food and Nutrition, Laboratory Study of Probiotics and Prebiotics, Faculty of \\ Pharmaceutical Sciences of Araraquara, UNESP-Paulista State University, Araraquara, São \\ Paulo,Brazil; ${ }^{2}$ School of Engineering of São Carlos, University of São Paulo, Sao Paulo, \\ Brazil
}

Corresponding author: Sivieri, Katia, PhD, Professor, Department of Food and Nutrition, Araraquara/Jau, Km 1, Araraquara, São Paulo,Brazil

Submission date: September 05, 2011; Acceptance date: October 07, 2011; Publication date: October 08, 2011

\section{$\underline{\text { Abstract }}$}

Background: Enterococci are used in a large number of dairy products, such as starter cultures in food supplements and in foods considered functional. In

vitro gut fermentation models present an unmatched opportunity of performing studies frequently allenged in humans and animals owing to ethical concerns. A dynamic model of the human intestinal microbial ecosystem (SHIME) was designed to better simulate conditions intestinal microbiota.

Methods: The SHIME model was used to study the effect of Enterococuus faecium CRL 183 on the fermentation pattern of the colon microbiota. Initially, an inoculum prepared from human feces was introduced into the reactor vessels and stabilized over 2 wk using a culture medium. This stabilization period was followed by a 2-wk control period during which the microbiota were monitored. The microbiota were then subjected to a 4-wk treatment period by adding $10^{8} \mathrm{CFU} / \mathrm{mL}$ of the Enterococcus faecium CRL 183 to vessel one (the stomach compartment).

Results: The addition resulted into an overall increase of bacterial marker populations (Enterobacteriaceae, Lactobacillus spp., Bifidobacterium spp. and Clostridium spp.), with a significant increase of the Lactobacillus sp. and Bifidobacterium sp populations. The shortchain fatty acid (SCFA) concentration increased during the supplementation period; this was due mainly to a significant increase in the levels of acetic, butyric and propionic acids. Ammonium concentrations increased during the supplementation period.

Conclusions: Results showed that the major effect of E. faecium CRL 183 was found in the ascendant and transverse colon. 
Key words: Gut microbiota, Enterococcus, Gastrointestinal resource management, Simulator of Human Intestinal Microbial Ecosystem (SHIME)

\section{BACKGROUND:}

The gastrointestinal (GI) tract is colonized by a vast community of symbionts and commensals that harbor a complex and diverse ecology of microorganisms comprised of 400500 species with levels reaching $10^{14} \mathrm{CFU} / \mathrm{g}$ of intestinal contents in the large intestine [1]. These microbes have far-reaching implications on health in that they affect immunity and digestion of nutrients. Microbial interactions contribute to the homeostasis of the gut bacterial microbiota and destabilization of this microorganism ecosystem results in various GI disorders [2]. It has been suggested that probiotics help to maintain the GI equilibrium of the indigenous microbiota and benefit the host's health. They are thus defined as "live microorganisms, that when administered in an adequate amounts, confer a health benefit on the host" [3]. Probiotic strains are considered as safe and non-pathogenic [4].

Enterococci are used in a large number of dairy products, such as starter cultures in food supplements and in foods considered functional [5]. Currently, many researchers seek microorganisms that have probiotic properties, as is the case of, for example, Enterococcus faecium CRL 183. Our research group verified that E. faecium CRL 183 has the capacity to survive in and colonize the gastrointestinal tract of rats [6], one of the prerequisites for being considered probiotic [7], since the viability of lactic bacteria can be lost on exposure to gastric acid and to bile salts [8]. Our group also observed that the consumption of 200 $\mathrm{mL}$ /day of soymilk fermented with E. faecium CRL 183 and Lactobacillus helveticus subsp. jugurti 416 by normocholesterolemic adult men, for a period of 6 weeks, reduced the levels of total cholesterol and of the LDL fraction and led to an increase of about $10 \%$ in HDL-C levels [9]. Others beneficial health effects, such as partial inhibition of breast cancer [10], prevention of cancer colon [11] and osteoporosis [12] have also been achieved with $E$. faecium CRL 183.

However, the influence of E. faecium consumption on human gut microbial fermentation has been little investigated to date. For that reason, the aim of our study was to investigate the effects of E. faecium CRL 183 on the fermentative capacity of the simulated microbiota of the colon.

\section{METHODS:}

\section{Preparation of the $E$. faecium CRL 183 cells:}

At weekly intervals, a pure culture of E. faecium CRL 183 was inoculated into MRS Agar culture medium (Acumedia, Baltimore). The bacteria in the log phase was centrifuged (4000 $\mathrm{x}$ g, $10 \mathrm{~min} ., 4^{\circ} \mathrm{C}$ ) and washed with sterile peptone water. The E. faecium cells were kept at the concentration of $10^{8} \mathrm{CFU} / \mathrm{mL}$ in sterile peptone water until use [6].

\section{Long-term SHIME run:}

The SHIME is a simulator of the human intestinal microbial ecosystem $[13,14]$ in which the environmental conditions ( $\mathrm{pH}$, residence time, inoculum, and temperature) are controlled to resemble those of in vivo experiments. A SHIME system consists of five double-jacketed vessels, respectively simulating the stomach, the small intestine, and the ascending, transverse and descending colon, with a total retention time of $72 \mathrm{~h}$ (Figure 1). Reactor setup 
and the composition of the liquid feed (Table 1), which entered the system 3 times per day were previously described by Possemiers et al. [15].

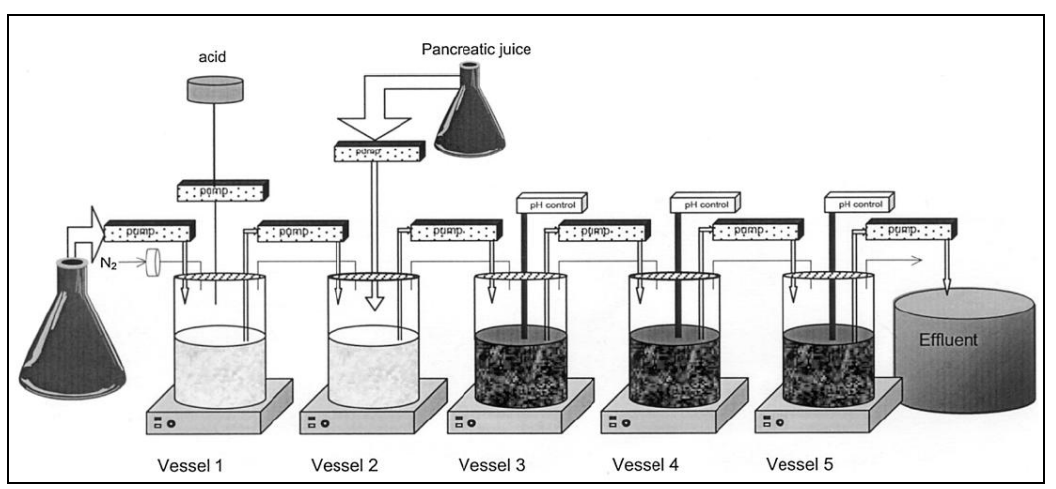

Figure 1. Schematic representation of the Simulator of the Human Intestinal Microbial Ecosystem (SHIME)

The three colon vessels of the SHIME reactor were inoculated with bacteria from a fecal sample of a healthy 22-year-old adult female volunteer with no history of antibiotic treatment 6 months prior to the study. Aliquots $(10 \mathrm{~g})$ of freshly fecal samples were diluted and homogenized with $100 \mathrm{~mL}$ sterilized phosphate buffer $(0.1 \mathrm{~mol} / \mathrm{L}, \mathrm{pH} 7)$, containing $1 \mathrm{~g} / \mathrm{L}$ sodium thioglycolate as reducing agent. After removal of the particulate material by centrifugation, the supernatants were pooled and $50 \mathrm{~mL}$ was introduced into each of the colon simulation vessels.

Table 1. Ingredients (g) for one liter of the basal feed utilized in Shime reactor

\begin{tabular}{lc}
\hline Ingredient & Quantity necessary for 1L \\
\hline Arabinogalactan & 1.0 \\
Pectin & 2.0 \\
Xilan & 1.0 \\
Potato starch & 3.0 \\
Glucose & 0.4 \\
Yeast extract & 3.0 \\
Peptone & 1.0 \\
Mucin & 4.0 \\
Cystein & 0.5 \\
Sterile distilled water & 1000 \\
\hline
\end{tabular}

The microbial inoculum was stabilized over a period of 2 weeks on a carbohydrate-based medium and allowed to adapt to the specific environmental conditions of the ascending, transverse and descending colon in terms of $\mathrm{pH}$ range, retention time and available carbon sources [15]. An initial stabilization period of two weeks after inoculation was applied to allow the intestinal bacteria to adapt to the environmental conditions present in the colon vessels and to form a stable microbial community representative of the one present in the gastrointestinal tract [20]. Upon stabilization, the SHIME run included 2 weeks of basal period (to quantify all steady-state bacterial parameters which were used as starting point to evaluate the effect of a specific treatment), 4 weeks of treatment period in which $3 \mathrm{~mL}$ of $10^{8}$ 
$\mathrm{CFU} / \mathrm{mL}$ of E. faecium CRL 183 were added once per day to the stomach compartment. Finally, a 2-week washout period without E. faecium addition.

\section{Microbiological analysis}

At weekly intervals, throughout the entire experimental period, (basal, treatment and washout), $5 \mathrm{~mL}$ - samples were collected from the reactors for microbiological examinations. Analysis of the composition of the intestinal microbiota was based on the enumeration of total aerobic and anaerobic bacteria, Enterococcus ssp., Lactobacillus ssp., Bifidobacterium ssp., Enterobacteria and, Clostridium ssp. One $\mathrm{mL}$ of a sample taken from each reactor was suspended into $99 \mathrm{~mL}$ peptone water. Serial dilutions were prepared and inoculated into selective culture media: total aerobic and anaerobic counts: - Standard Methods Agar (Acumedia, USA; $37^{\circ} \mathrm{C} / 48 \mathrm{~h}$ ); Enterococcus spp.: - KF Streptococcus Agar (Acumedia, USA; 37 ${ }^{\circ} \mathrm{C} / 48 \mathrm{~h}$ ) [16]; Lactobacillus spp.:- MRS Agar (Merck, Germany; 37 ${ }^{\circ} \mathrm{C} / 48 \mathrm{~h}$, under anaerobiosis); Bifidobacterium spp.:- Bifidobacterium formulated medium BIM-25 (supplier, $37^{\circ} \mathrm{C} / 72 \mathrm{~h}$, under anaerobiosis) [17], Enterobacteria:- MacConkey Agar (Acumedia, USA; $37^{\circ} \mathrm{C} / 48 \mathrm{~h}$ ) and Clostridium spp.: RCA Agar (Difco, France; $37^{\circ} \mathrm{C} / 48 \mathrm{~h}$, under anaerobiosis) [18].

\section{Analysis of short-chain fatty acid and ammonium}

Once a week, throughout the entire experimental period (basal, treatment and washout), samples were collected from the reactors for analysis of short-chain fatty acids (SCFA) and ammonium. The analysis was carried in triplicate.

Every week, the levels of short-chain fatty acids (SCFA) were determined from samples collected from the reactors and frozen to $-20^{\circ} \mathrm{C}$. The SCFA were extracted with diethyl ether and determined using a gas chromatograph equipped with a flame-ionization gas detector, a capillary split/splitless injector and an HP-INNOWAX column with a $30 \mathrm{~m} \times 0.25$ $\mathrm{mm} \times 0.25 \mu \mathrm{m}$ inlet (Shimadzu GC2010), using hydrogen as carrier gas at a flow rate of 1.56 $\mathrm{mL} / \mathrm{min}$. The temperatures of the column, injector and detector were 170,250 and $280{ }^{\circ} \mathrm{C}$, respectively $[19,20]$.

The ammonia content was determined using a selective ion meter $(710 \mathrm{~A}$ model, Orion) coupled to an ammonia selective-ion electrode (Orion 95-12). The apparatus was calibrated using $0.1 \mathrm{M}$ standard ammonium chloride solutions, at the concentrations of 10 , 100 and $1000 \mathrm{mg} / \mathrm{L}$ ammonia. Each $25 \mathrm{~mL}$ of sample was added with $0.5 \mathrm{~mL}$ ISA solution (a $\mathrm{pH}$-adjusting Ionic Strength Adjuster (Orion) - a pH-adjusting and an ionic force solution). All measurements were taken at $25^{\circ} \mathrm{C}$ [21].

\section{Statistical analysis}

Significance of all results was investigated using the statistical software Sigma Stat 5.0. with one-way ANOVA, and individual means were compared using the Tukey's test.

\section{RESULTS AND DISCUSSION:}

\section{Microbiological evaluation}

It is estimated that the gastrointestinal microbiota harbors around $10^{14}$ bacteria. This microbiota undergoes both qualitative and quantitative changes depending on the locale of colonization. The huge complexity of this microbiota, which are often unculturable microorganisms (30 to $70 \%$ ) in culture media, in addition to being located in difficult-to- 
access areas of the digestive tract, which would require invasive methods to collect them, are the limiting factors for a more precise analysis [22].

An investigative alternative is the use of continuous or semi-continuous models simulating the large intestine. The continuous models was validated based on the intestinal contents of sudden death victims [23]. Among the advantages of this model are the ease-ofuse, the possibility to use radioactive substances and the low cost [24].

Table 2 shows the microbiological counts of the flasks that simulated the ascendant, transverse and descendant colon of the SHIME reactor. Using selective growth media, the microbiological analyses revealed the influence of the administration of E. faecium CRL 183 on the composition of the intestinal microbial community.

With regard to the enterobacterial population, no quantitative change was observed during the treatment period. However, Bedani et al., [25] observed a significant increase in the numbers of these microorganisms in the feces of rats that had consumed pure cells of $E$. faecium CRL 183 during 30 days.

Table 2. Average plate count measurements $( \pm \mathrm{SEM})$, expressed in $\log \mathrm{CFU} / \mathrm{mL}$, for the different microbial groups, SHIME compartments and periods

\begin{tabular}{llll}
\hline Bacterial groups & $\begin{array}{l}\text { Colon } \\
\text { ascendans }\end{array}$ & $\begin{array}{l}\text { Colon } \\
\text { transversum }\end{array}$ & $\begin{array}{l}\text { Colon } \\
\text { descendans }\end{array}$ \\
\hline Basal & & & \\
Enterococcus spp. & $6.62^{\mathrm{A}} \pm 0.47$ & $7.37^{\mathrm{A}} \pm 0.05$ & $8.27^{\mathrm{A}} \pm 0.03$ \\
Enterobacteria & $7.30^{\mathrm{A}} \pm 0.14$ & $6.23^{\mathrm{A}} \pm 0.08$ & $6.53^{\mathrm{A}} \pm 0.08$ \\
Lactobacillus spp. & $6.95^{\mathrm{A}} \pm 0.05$ & $4.13^{\mathrm{A}} \pm 0.22$ & $7.68^{\mathrm{A}} \pm 0.09$ \\
Bifidobacterium spp. & $6.88^{\mathrm{A}} \pm 0.05$ & $6.08^{\mathrm{A}} \pm 0.12$ & $9.25^{\mathrm{A}} \pm 0.03$ \\
Clostridium spp. & $7.72^{\mathrm{A}} \pm 0.01$ & $7.80^{\mathrm{A}} \pm 0.01$ & $7.72^{\mathrm{A}} \pm 0.02$ \\
Total aerobes & $7.63^{\mathrm{A}} \pm 0.04$ & $7.16^{\mathrm{A}} \pm 0.01$ & $7.66^{\mathrm{A}} \pm 0.01$ \\
Anaerobes facultative & $6.68^{\mathrm{A}} \pm 0.10$ & $7.22^{\mathrm{A}} \pm 0.07$ & $7.68^{\mathrm{A}} \pm 0.0$ \\
Treatment & & & \\
Enterococcus spp. & $8.90^{\mathrm{B}} \pm 0.77$ & $9.06^{\mathrm{B}} \pm 0.54$ & $8.72^{\mathrm{A}} \pm 0.54$ \\
Enterobacteria & $7.00^{\mathrm{A}} \pm 0.27$ & $7.11^{\mathrm{A}} \pm 0.83$ & $6.96^{\mathrm{A}} \pm 0.93$ \\
Lactobacillus spp. & $8.54^{\mathrm{B}} \pm 1.54$ & $8.25^{\mathrm{B}} \pm 1.64$ & $8.50^{\mathrm{A}} \pm 0.54$ \\
Bifidobacterium spp. & $9.40^{\mathrm{B}} \pm 0.82$ & $8.74^{\mathrm{B}} \pm 0.94$ & $9.50^{\mathrm{A}} \pm 0.66$ \\
Clostridium spp. & $8.88^{\mathrm{A}} \pm 1.33$ & $8.59^{\mathrm{A}} \pm 1.52$ & $8.71^{\mathrm{A}} \pm 1.43$ \\
Total aerobes & $8.45^{\mathrm{A}} \pm 1.80$ & $8.58^{\mathrm{A}} \pm 1.04$ & $8.22^{\mathrm{A}} \pm 0.07$ \\
Anaerobes facultative & $8.82^{\mathrm{B}} \pm 0.53$ & $8.90^{\mathrm{B}} \pm 0.56$ & $8.85^{\mathrm{B}} \pm 0.56$ \\
Washout & & & \\
Enterococcus spp. & $7.46^{\mathrm{AB}} \pm 0.22$ & $7.01^{\mathrm{A}} \pm 0.64$ & $7.24^{\mathrm{B}} \pm 0.31$ \\
Enterobacteria & $5.37^{\mathrm{B}} \pm 0.07$ & $5.79^{\mathrm{A}} \pm 0.51$ & $5.66^{\mathrm{B}} \pm 0.25$ \\
Lactobacillus spp. & $7.28^{\mathrm{B}} \pm 0.02$ & $7.13^{\mathrm{B}} \pm 0.02$ & $7.08^{\mathrm{A}} \pm 0.01$ \\
Bifidobacterium spp. & $7.99^{\mathrm{A}} \pm 0.06$ & $7.88^{\mathrm{CB}} \pm 0.89$ & $8.00^{\mathrm{B}} \pm 0.09$ \\
Clostridium spp. & $7.79^{\mathrm{A}} \pm 0.45$ & $7.54^{\mathrm{A}} \pm 0.62$ & $7.56^{\mathrm{A}} \pm 0.50$ \\
Total aerobes & $7.86^{\mathrm{A}} \pm 0.06$ & $7.85^{\mathrm{A}} \pm 0.14$ & $7.71^{\mathrm{A}} \pm 0.08$ \\
Anaerobes facultative & $8.08^{\mathrm{AB}} \pm 0.53$ & $8.05^{\mathrm{AB}} \pm 0.03$ & $7.97^{\mathrm{AC}} \pm 0.02$ \\
\hline
\end{tabular}

Different letters indicate significantly different results $(\mathrm{P}<0.05)$ in same microbial group and same compartment (colon ascendans, transversum or descendans). 
In previous studies conducted by our research group [26], no quantitative change was observed in the microbiota of Enterococcus spp contained in the feces of rats that had been daily fed with E. faecium CRL 183 during 30 days. However, in the SHIME reactor, during the treatment period, a statistically significant increase in the Enterococcus ssp counts occurred in the ascendant ( 1 log cycle) and transverse ( 2 log cycles) colons. Probably, E. faecium CRL 183 has a greater capacity to colonize and adhere to the ascending and transversal regions of the colon. According to Jin et al [27], E. faecium occupies binding sites in the mucosal cells of the ascendant colon, which allow their adherence.

The concentration of the Lactobacillus ssp population increased significantly by two logarithmic cycles in the ascendant colon and by three log cycles in the transverse colon during the treatment phase. However, in the descendant colon, there was no statistically significant alteration during the same phase. According to Marteau [28], the colon is the primary microbial colonization site, a region consisting of different niches and ecosystems. The $\mathrm{pH}$ in the ascending colon is about 5.6 to 5.9, a value that favors the growth of Lactobacillus ssp. According to Coudeyras and Forestier [29], the ascendant and transverse regions of the colon harbor a microbiota that is very similar to that of the stomach, with predominance of facultative aerobes and anaerobes. The dominant genus in this region is Streptococcus ssp, but Lactobacillus ssp. and Enterococcus ssp also being found here, both of which are species that are generally present in the intestinal lumen contents. The results show that the administration of E. faecium stimulated the growth of Lactobacillus ssp in the ascendant and transverse colons.

In the descendant colon, the population of bifidobacteria accounts for about 3 to 5\% of the total microbiota in this region [29]. Over the basal period, a higher count was observed $\left(10^{8} \mathrm{CFU} / \mathrm{mL}\right)$ in the descending colon, as compared to the ascendant and transverse regions $\left(10^{5} \mathrm{UFC} / \mathrm{mL}\right)$. However, the treatment with E. faecium stimulated the growth of the population of bifidobacteria only in the ascending and transverse colon regions. Bedani et al., [25] observed that the animals that received a suspension of a pure E. faecium CRL 183 culture presented an increase in the fecal bifidobacteria population in the feces.

As for the population of Clostridium spp., there was no statistical significant difference during the treatment period in the three regions of the colon evaluated. Bacteria belonging to this genus may be harmful due to their metabolic activity and the pathogenic character of some species [30]. The species belonging to the Clostridium genus may be involved in inflammatory processes of intestinal diseases [31]. Bedani et al, [25] also observed that there was no alteration in the fecal population of Clostridium ssp in rats that consumed cultures of E. faecium CRL 183.

With regard to total aerobes, there was no statistically significant change in the population of these microorganisms during the treatment period. As for the facultative aerobes, a significant increase was noted in the ascendant, transverse and descendant colons. According to Coudeyras and Forestier [29], one of the main differences between the microbiota found in the feces and that in the colon is related to the facultative anaerobes, which are abundant in the colon and practically absent from the feces.

A comparison between the basal, treatment and washout periods allows to state that, in a general manner, all the microbial groups evaluated had their concentrations reduced in the post-treatment period. According to Doré and Corthier [32], the dominant intestinal microbiota is resistant to modifications. The administrations of probiotics or prebiotics may temporarily change intestinal homeostasis. Furthermore, it may be stated that E. faecium CRL 
183 stimulate the growth of some microbial groups, such as; Lactobacillus ssp., and Bifidobacterium ssp in the ascendant and transverse regions of the colon, exerting little influence in the descending region.

\section{Ammonium concentration}

The intestinal microbiota is a complex ecosystem composed of interdependent bacteria. Certain bacteria stabilize themselves solely as a function of metabolites produced by other bacteria, and together they transform complex polymers into simple molecules [29].

The final metabolites from the digestion of glycides by the intestinal bacteria are short-chain fatty acids, $\mathrm{H}_{2}$ and $\mathrm{CO}_{2}$. On the other hand, the degradation products of proteins are short-chain fatty acids, branched fatty acids, phenolic derivatives, indolic derivatives, polyamines and ammonia. The deleterious metabolites are amines and ammonia. These metabolites may be either absorbed by the body or excreted through urine [28]. Studies have demonstrated that high concentrations of ammonia act as tumor-promoting agents in the colon, since they are toxic to the epithelial cells of the intestine [22].

Figure 2 shows the concentration of ammonium ion in the basal, treatment and washout phases.

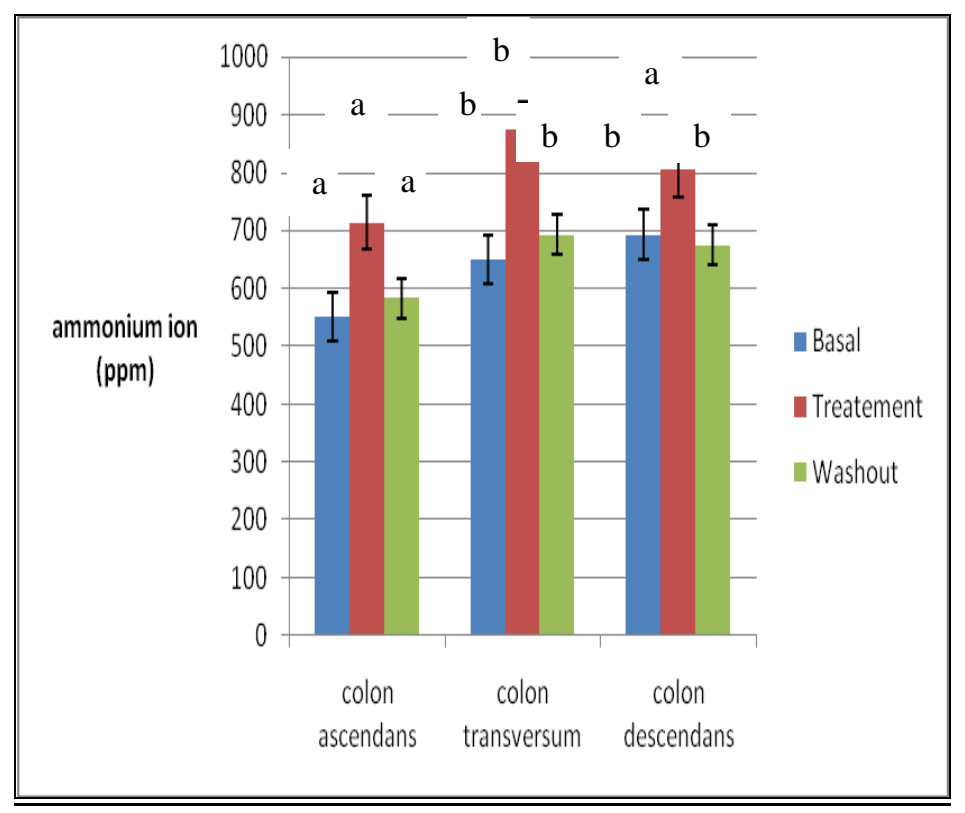

Figure 2. Average ammonium ion production ( $\mathrm{ppm}$ ) in shime run, during basal, treatment and washout period. Statistically significant differences among the samples were investigated with one-way ANOVA (samples with the same letter on the top of the bar are not statistically different, $P<0.05)$.

During the treatment period with E. faecium, the concentration values of concentration of ammonium ion increased significantly in all the regions investigated (Figure 2). Similar results were observed by Bedani et al [21], who noted a significant increase in the concentration of ammonia in that had been fed daily doses of E. faecium CRL 183 cells. Pomessier et al., [15] and Payne et al., [33] also observed an increase in the ammonia content in an experiment utilizing a dynamic simulator of the human microbial ecosystem.

Urease is expressed by many bacteria and mediated by the hydrolysis of urea into ammonia, which serves a source of bacterial nitrogen. According to Laukoová and Kuncová 
[34], some strains of E. faecium have urease activity, which would explain the increase in the concentration of ammonia during the administration of E. faecium cells.

\section{Fermentation capacity}

One of the most important factors for the persistence of the intestinal microbiota is the diet, which does not only provides the host with nutrients, but also the intestinal microorganisms [18]. In this sense, the carbohydrates that are not digested in the colon are metabolized by microorganisms into SCFA, particularly acetate, butyrate and propionate. The formation of short-chain fatty acids is of great importance, since they are sources of energy and serve as microbial substrate, in addition to being related to several organic, local and systemic effects [35]. The production of SCFA depends on the substrates available and the microorganisms present in the gastrointestinal tract [36].

Figure 3 depicts the production of acetate during the periods of basal, treatment and washout in the Shime vessels. During treatment with E. faecium, a significant increase occurred in the production of acetate in all the reactors analyzed, however, the greatest concentration of this acid occurred in vessel one, which simulates the ascending region of the colon. In the washout period, the levels of acetate diminish, however, only in the transverse colon these levels differ statistically from the basal period.

A significant increase in the concentration of butyrate was observed in the vessels simulating the transverse and descendant colon (Figure 4). Butyrate is considered the preferred fuel of the epithelial cells of the colon, which derive $70 \%$ of their energy from the oxidation of this substrate. Butyrate also reduces the expression of proinflammatory cytokines of tumor necrosis factor- $\alpha$ (TNF- $\alpha$ ), TNF- $\beta$, interleukine-6 (IL-6) and IL-1 $\beta$ through activation of the nuclear growth inhibiting factor $\mathrm{kB}$ (NF-kB) (23). In addition, it has been proposed that butyrate reduces the risk of colon cancer due to is ability to inhibit the genotoxic capacity of nitrosamines and of hydrogen peroxide, as well as to induce different levels of apoptosis, differentiation and cessation of the cellular cycle of colon cancer in animal models [36].

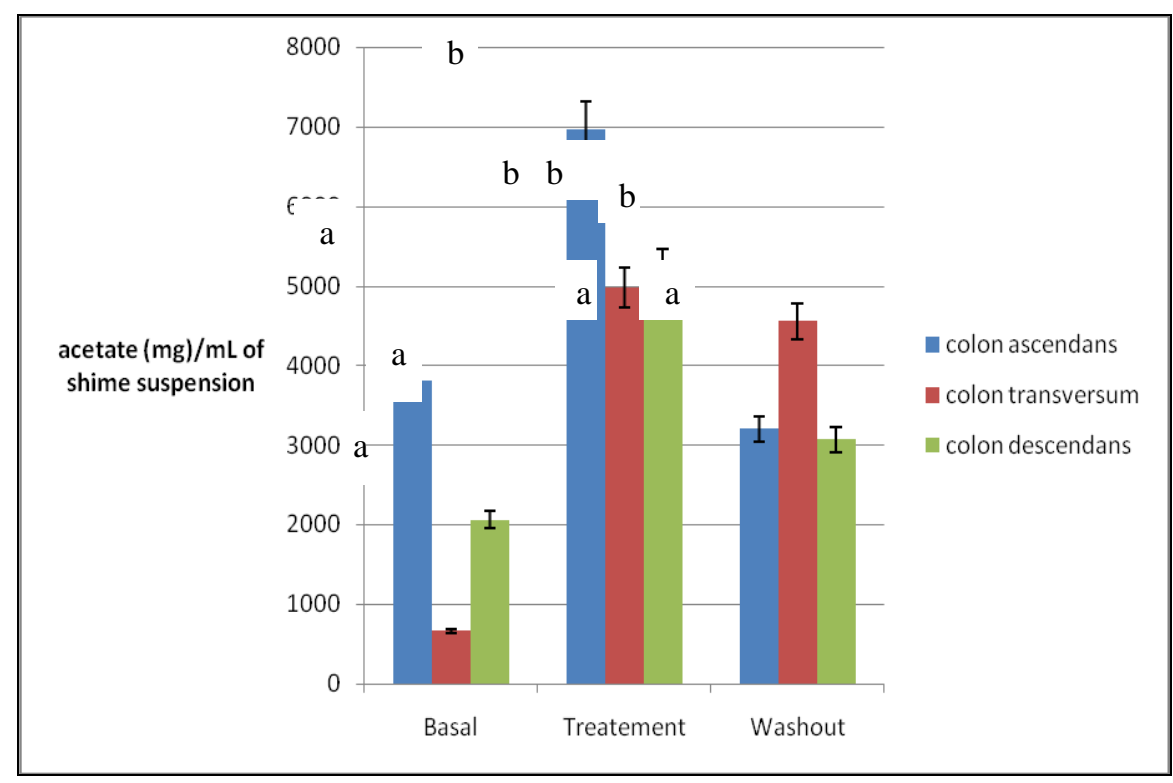

Figura 3. Average SFCA production (acetate) during the SHIME run, in the basal, treatment and washout period, respectively. Statistically significant differences among the samples were 
investigated with one-way ANOVA (samples with the same letter on the top of the bar are not statistically different, $P<0.05$ )

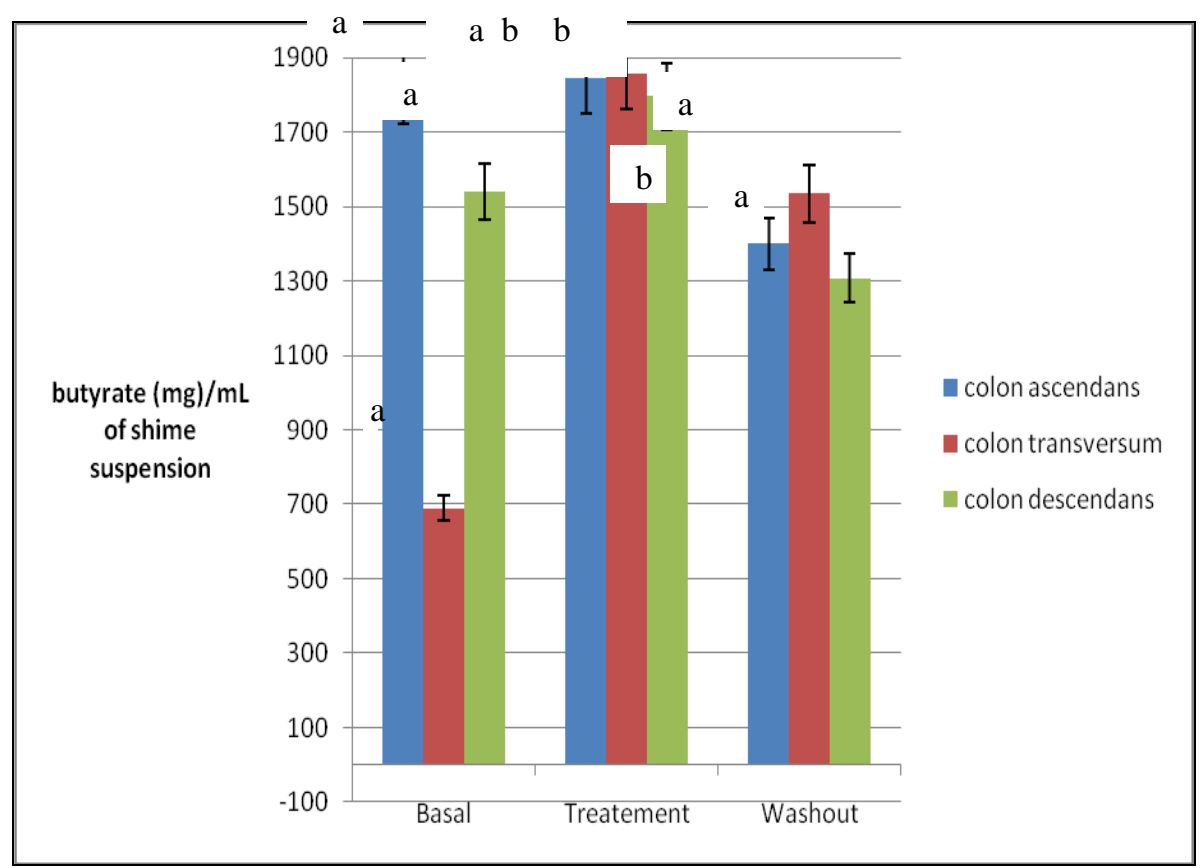

Figura 4. Average SFCA production (butyrate) during the SHIME run, in the basal, treatment and washout period, respectively. Statistically significant differences among the samples were investigated with one-way ANOVA (samples with the same letter on the top of the bar are not statistically different, $P<0.05$ )

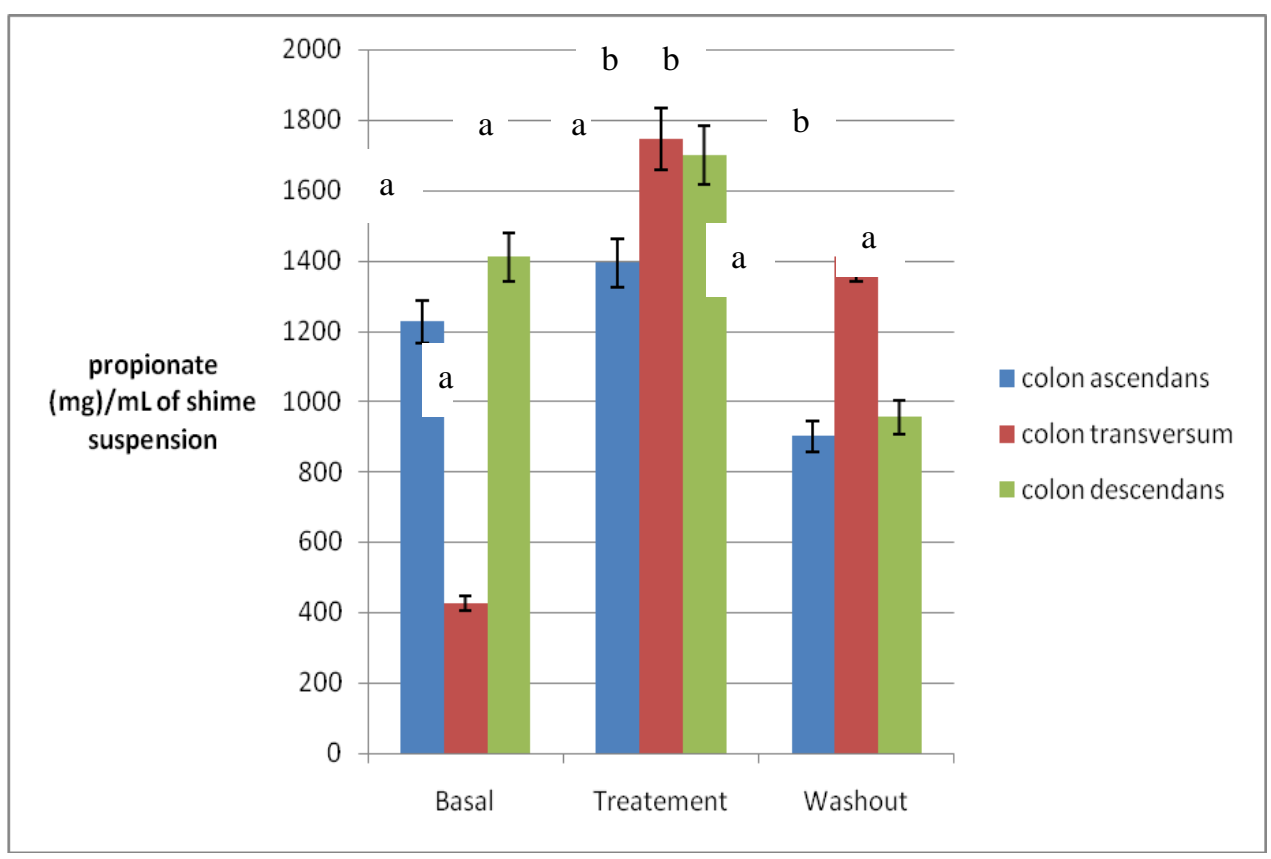

Figure 5. Average SFCA production (propionate) during the SHIME run, in the basal, treatment and washout period, respectively. Statistically significant differences among the samples were investigated with one-way ANOVA (samples with the same letter on the top of the bar are not statistically different, $P<0.05$ ) 
Butyrate has been studied in clinical applications, particularly inflammatory bowel diseases of the colon. While available data are not entirely conclusive, this substance appears to have a useful therapeutic role complementary to that of standard drugs. A body of experimental data also suggests that this short-chain fatty acid may exert preventive action against colorectal cancer, but for the moment this is still a hypothesis that remains to be verified. More generally, butyrate has been shown to be useful in certain types of diarrhea, particularly chronic forms, by promoting absorption of water and electrolytes [37].

Previous studies conducted using an animal model allowed to observe that the consumption of pure cells of E. faecium CRL 183 inhibited the development of colon cancer [11]. Within this context, one may assume that this inhibition may be connected with the production of butyrate observed in in vitro experiments.

With regard to propionate, a significant increase was observed in the concentrations in the vessels 2 and 3, that simulate the transverse and descendant colon during the treatment with E. faecium. In the washout period, a reduction in the concentration of this acid was found to have occurred, however only in the descendant colon this variation was statistically significant (Figure 5).

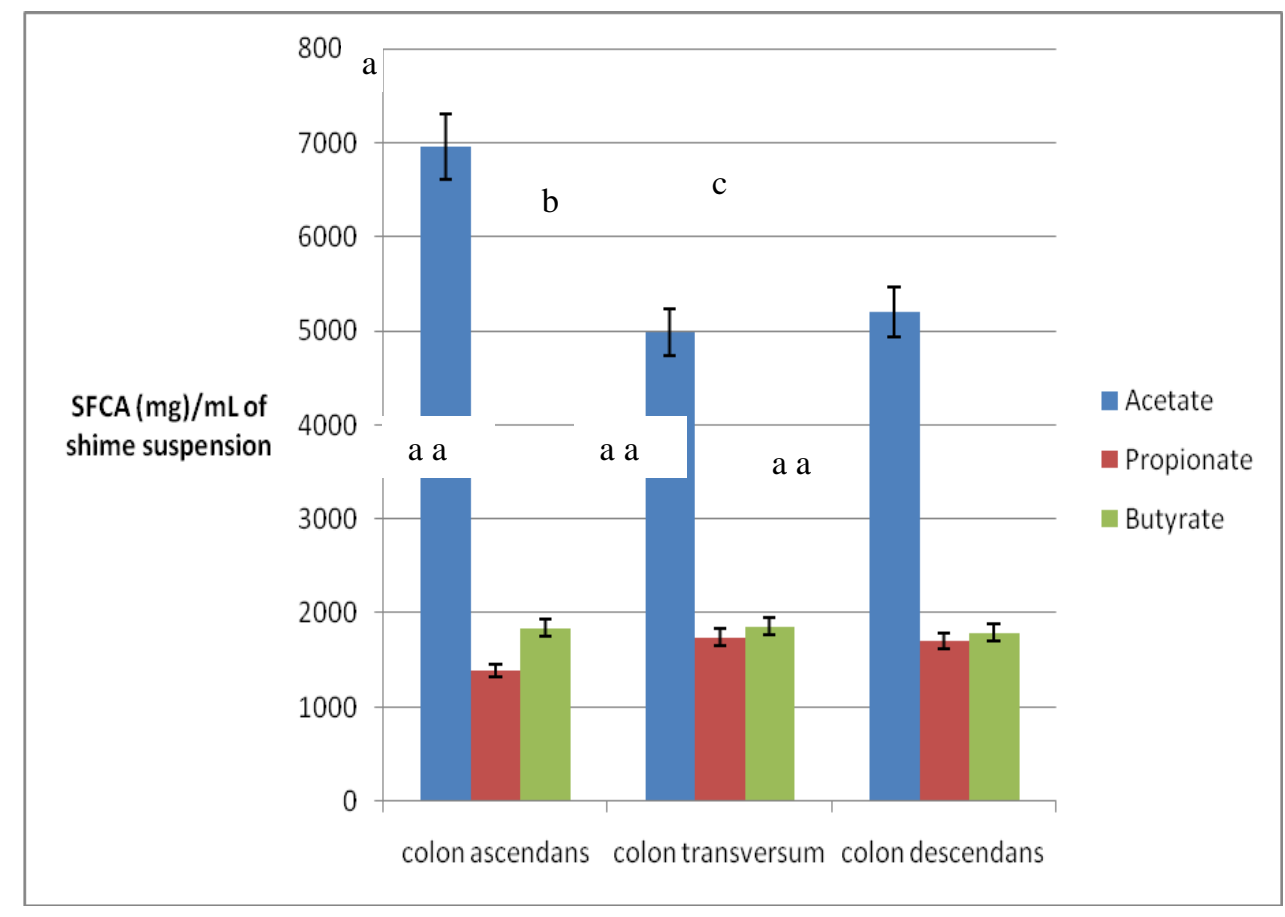

Figure 6. Average SFCA production during the SHIME run in the treatment period. Statistically significant differences among the samples were investigated with one-way ANOVA (samples with the same letter on the top of the bar are not statistically different, $P<0.05)$

Figure 6 refers to the production of SCFA (acetate, propionate and butyrate) during the treatment phase in the reactors that simulate the ascendant, transverse and descendant colon. The acid with the greatest production was acetate. Comparing the three compartments of the colon, it is observed that there was a significant difference between the ascendant colon and the transverse and descending colons for this acid. As for the other acids investigated (butyrate and propionate) there were no significant differences in the concentrations between the regions of the colon. The same pattern was observed in the washout period, that is, upon 
cessation of the treatment with E. faecium, the production of SCFA diminished, but the tendency between the regions of the colon remained unchanged (Figure 7). Similar results were observed by Van de Wiele et al., [19] in experiments using the Shime reactor.

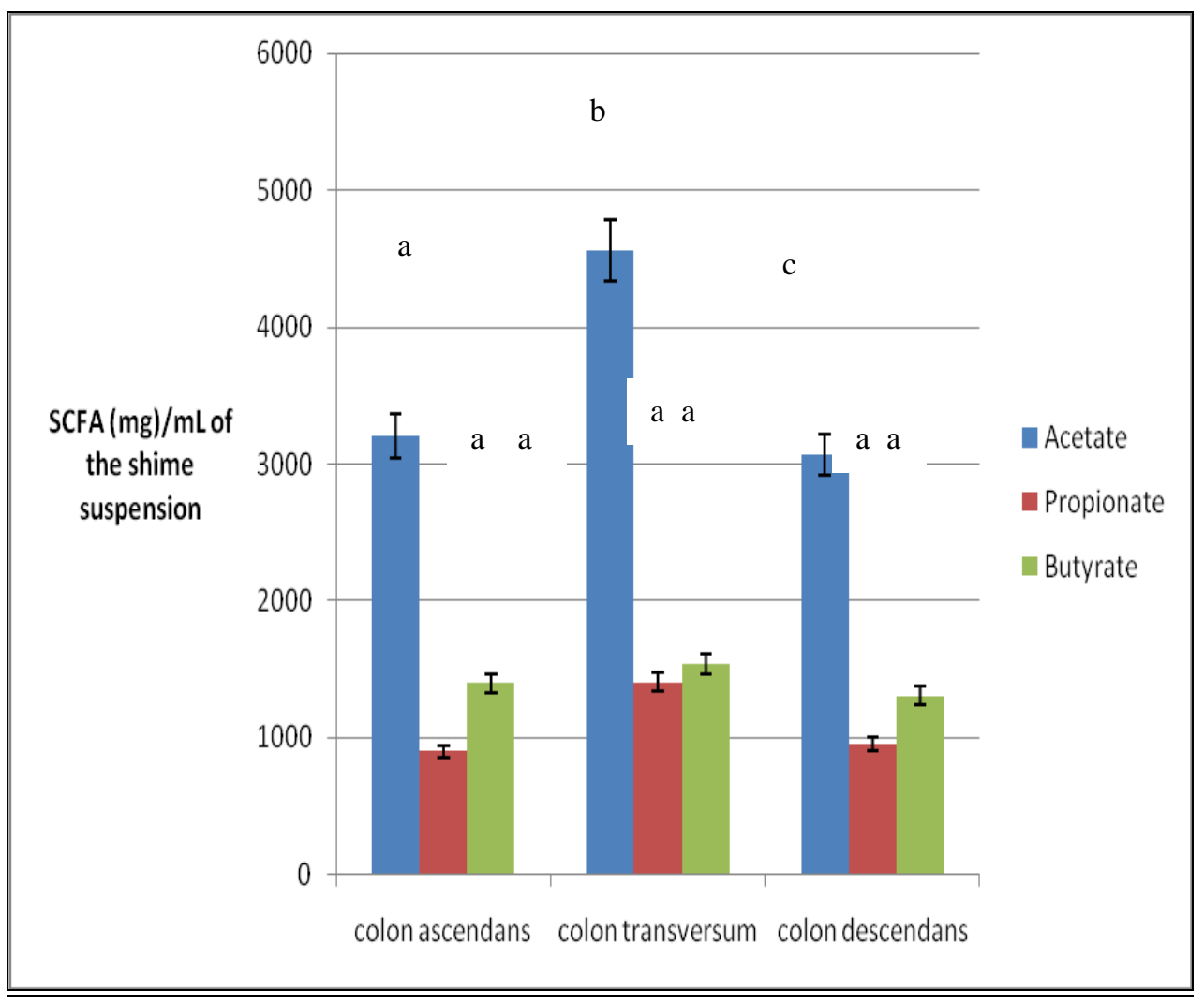

Figura 7. Average SFCA production during the SHIME run in the washout period. Statistically significant differences among the samples were investigated with one-way ANOVA (samples with the same letter on the top of the bar are not statistically different, $P<0.05)$

The production of SCFA in the treatment period may be explained by the increase in the concentration of lactobacilli and bifidobacteria observed over the same period of time (Table 2), which use the breakdown of the starch present in the basal medium with the consequent production of SCFA. According to Van de Wiele et al., [20] the increase in the synthesis of fatty acids creates a more acid intestinal environment, which is important for the colonization resistance against potentially pathogenic microorganisms. On the other hand, the SCFA are important sources for the colonocytes, in addition to stimulating the absorption of water and sodium and modulating intestinal motility [38].

This study indicates that the consumption of E. faecium may influence the gut microbiota in a beneficial way. The concentration of SCFA increased, whereas a concentration of the harmful putrefactive metabolite $\left(\mathrm{NH}_{3}\right)$ was also augmented. Apparently, E. faecium had greater influence on the gut microbiota of the ascendant and transversal colon. Limitations associated with in vitro systems [39] also should trigger further research to study the beneficial effects of E. faecium consumption in vivo.

Abbreviations: short-chain fatty acids (SCFA), Simulator of the human intestinal microbial ecosystem (SHIME), gastrointestinal (GI); 
Competing interests: No competing interests.

Acknowledgements: This work was supported by Fundação de Amparo à Pesquisa do Estado de São Paulo (FAPESP) (2009/53878-8).

\section{Authors Contributions}

Sivieri, Katia, $\mathrm{PhD}$ is the principle investigator for this study providing oversight and contributed fundamental conceptualization for the research, writing a grant proposal and manuscript. Bianchi, Fernanda, performed the microbiological analysis for the study, Rossi, Antonio Elizeu, $\mathrm{PhD}$ is the research coordinator for the study. Adorno, Maria Angela Tallarico, performed the SCFA analysis.

\section{REFERENCES}

1. Berg RD. The indigenous gastrointestinal microflora. Trends Microbiol 1996; 4: 430-435.

2. Raibaud P. Bacterial interations in the gut. In: Fuller R., editor. Probiotics: the scientific basis. London: Chapman \& Hall; 1992. p. 9-28.

3. Food and Agriculture Organization of United Nations; World Health Organization. FAO/WHO (2001). Available from. Evaluation of health and nutritional properties of probiotics in food including powder milk with live lactic acid bacteria. Report of a joint FAO/WHO expert consultation, Cordoba, Argentina. $\mathrm{ftp}$ //ftp.fao.org/es/esn/food/probioreport_en.pdf

4. Salminen S, Von Wright A, Morelli L. Demonstration of safety of probiotics a review. Int J Food Microbiol 1998; 44: 93-106.

5. Klein G. Taxonomy, ecology and antibiotic resistance of enterococci from food and the gastro-intestinal tract. Int J Food Microbiol 2003; 88:123-131.

6. Sivieri K, Cano VS, Valentini SR, Rossi EA. Demonstration of the cellular viability and safety of Enterococcus faecium CRL 183 in long-term experiments. Le Lait 2007; 87: 59-69

7. Lund B, Admsson I, Edlund C. Gastrointestinal transit survival of an Enterococcus faecium probiotic strain administered with or without vancomycin. Int J Food Microbiol 2002; 77:109-115.

8. Bezkorovainy A. Probiotics: determinants of survival and growth in the gut. Am J Clin Nutr 2001;73:399-405

9. Rossi EA, Vendramine RC, Carlos IZ, Oliveira MG, Valdez GF. Efeito de um novo produto fermentado de soja sobre lípides séricos de homens Adultos normocolesterolêmicos. Arch Latinoam Nutr 2003; 53: 47-51.

10. Kinouchi F.L. (2006). Iogurte de soja como coadjuvante no tratamento de câncer de mama. Araraquara, 85p. (Tese, UNESP).

11. Sivieri K, Spinardi-Barbisan ALT, Barbisan LF, Bedani R, Pauly ND, Carlos IZ, Benzatti RC, Vendramini RC, Rossi EA. Probiotic Enterococcus faecium CRL 183 inhibit chemically induced colon câncer in male Wistar rats. Eur Food Res Technol 2008; 228: 331-337.

12. Bedani R, Rossi EA, Lepera JS, Wang CC, Valdez CF. Efeito de um novo produto fermentado de soja, enriquecido com isoflavonas e cálcio, sobre o tecido ósseo de ratas. Arch Latinoam Nutr 2006; 56:146-152. 
13. Molly K, Woestyne MV, Verstraete W. Development of a 5-step multichamber reactor as a Simulation of the Human Intestinal Microbial Ecosystem. Appl. Microbiol. Biotechnol 1993; 39:254-258.

14. Molly K, Vandewoestyne,M, Desmet I, Verstraete,W. Validation of the simulator of the human intestinal microbial ecosystem (SHIME) reactor using microorganism associated activities. Microb Ecol Health Dis 1994; 7:191-200.

15. Possemiers S, Verthé K, Uyttendaele,S.;Verstraete,W. PCR-DGGE-based quantification of stability of the microbial community in a simulator of the human intestinal microbial ecosystem. FEMS Microbiol Ecol 2004; 49:495-507.

16. Edlund C, Beyer G, Hiemer-Bau M, Ziege S, Lode H, Nord CE. Comparative effects of mixifloxacin and clrithromycin on normal intestinal microflora. Scand. J. Infect. Dis 2000; 32: 81-85.

17. Munoa FJ, Pares R. Selective medium for isolation and enumeration of Bifidobacterium spp. Appl Environ Microbiol 1998; 54: 1715-1718.

18. Marzotto M, Maffeis C, Paternoster T, Ferrrario R, Rizzotti L, Pellegrino M, Dellaglio F, Torriani S. Lactobacillus paracasei: A survives gastrointestinal passage and affects the fecal microbiota of healthy infants. Res Microbiol 2006;157: 857-866.

19. Van De Wiele T, Boon N, Possemiers S, Jacobs H, Verstraete W. (2007). Inulin-type fructans of longer degree of polymerization exert more pronounced in vitro prebiotic effects. J Appl Microbiol 2007; 102:452-460.

20. Van De Wiele, T Boon, N Possemiers, S Jacobs, H Verstraete. Prebiotic effect of chicory inulin in the simulator of the human intestinal microbial ecosystem. FEMS Microbiol Ecol 2004; 51:143-153.

21. Bedani R. (2008). Influência do consumo de "iogurte” de soja fermentado com enterococcus faecium CRL 183 na microbiota intestinal de animais e humanos. Araraquara, São Paulo, Brasil, 140p (M.Sc. Dissertation.Faculdade de Ciências Farmacêuticas,. UNESP).

22. Montalto M, D'onofrio F, Gallo A, Cazzato A, Gasbarrini G. (2009). Intestinal microbiota and its functions. Digest Liver Dis 2009; 3:30-34.

23. Macfarlane GT, Macfarlane S. Models for intestinal fermentation: association between food components, delivery systems, biovailability and functional interactions in the gut. Curr Opin Biotechnol 2007, 18: 156-162.

24. Gibson GR, Fuller R. Aspects of in vitro and in vivo research approaches directed toward identifying probiotics and prebiotics for human use. J. Nutr $2000 ; 130: 391-395$.

25. Bedani R, Silveira NPD, Roselino MN, Vaaldez GF, Rossi EA. (2010). Effect of fermented soy product on the fecal microbiota of rats fed on a beef-based animal diet. J Sci Food Agric 2010; 90: 233-238.

26. Sivieri K, Cano VS, Valentini SR, Rossi EA. Demonstration of the cellular viability and safety of Enterococcus faecium CRL 183 in long-term experiments. Le Lait 2007; 87: 59-69.

27. Jin LZ, Marquardt RR, Zhao X. A strain of Enterococcus faecium (18C23) inhibits adhesion of enteroxigenic Escherichia coli K88 to porcine small intestine mucus. Appl Environ Microbiol 2000; 66:4200-4204.

28. Marteau P. (2010). L'importance clinique du microbiote intestinalThe clinical importance of intestinal microbiota. Gastroenterol Clin Biol 2010; 34:99-103.

29. Coudeyras S, Forestier C. Microbiote et probiotiques: impact en santé humaine. Can J Microbiol 2010; 56:611-650. 
30. Montesi A, Garcia-Albiach R, Pozuelo MJ, Pintado C, Goni I, Rotger R. Molecular and microbiological analysis of caecal microbiota in rats fed with diets supplemented either with prebiotics and probiotics. Int J Food Microbiol 2005; 98:281-289.

31. Guarner F, Malagelada JR. (2003). Gut flora in health and disease. Lancet 2003; 361:512519.

32. Doré J, Corthier G. The human intestinal microbiota Le microbiote intestinal humain. Gastroenterol Clin Biol 2010, 34:S7-S15.

33. Payne S, Gibson G, Wynne A, Hudspith B, Brostoff J, Tuohy K. In vitro studies on colonization resistance of the human gut microbiota to Candida albicans and the effects of tetracycline and Lactobacillus plantarum LPK. Curr. Issues Intest. Microbiol 2003; 4:1-8.

34. Laukoyá A, Kuncová M. Lactic acid production and urease activity in strains of Enterococcus faecium found in the rumen and their genetic stability. Vet Med 1991; 36:335340.

35. Cummings JH, Macfarlane GT. Gastrointestinal effects of prebiotics. Br J Nutr 2002; 87:145-51.

36. Bedani R, Rossi EA. Microbiota intestinal e probióticos: implicações sobre o câncer de cólon. J Port Gastrenterol 2009; 15:19-28.

37. Vernia P. (2008). Butyrate in the treatment of ulcerative colitis. Digest Liver Dis 2008; 1: 2730.

38. Cherbut C, Aube AC, Blottiere HM, Galmiche JP. (1997). Effects of short-chain fatty acids on gastrointestinal motility. Scand J Gastroenterol 1997; 32:58-61.

39. Payne AN, Zihler A, Chassard C, Lacroix C. Advances and perspectives in in vitro human gut fermentation modeling. Trends in Biotechnol 2011, (on line version). 